\title{
L'inégalité économique, sociale et psychique des femmes: une question démocratique
}

\author{
Claudine Haroche ${ }^{36}$
}

Resumé: On s'inscrit ici dans une approche sociologique et historique voulant discerner la part de l'anthropologique et du politique dans l'inégalité entre les hommes et les femmes. Certaines des observations visionnaires de Tocqueville portant sur les relations entre homme et femme, la comparaison qu'il en fait entre l'Amérique et la France, contribue à élucider en profondeur les relations entre hommes et femmes. On en vient ensuite à l'analyse de faits contemporains et on réexamine enfin en conclusion les causes lointaines et les formes - insidieuses en particulier - de cette dévalorisation persistante des femmes.

Mots-clés: Inégalités entre hommes et femmes, Démocratie, Dépendance économique, Privilège masculin, Domination insidieuse.

Dans le passé, il a fallu attendre les travaux des féministes, - qu'on parle de l'existence d'un sexe faible et d'un sexe fort - pour qu'on prenne conscience de la dépendance économique, sociale, politique, privée des femmes, qu'on en explicite la teneur, qu'on ne cesse d'exiger des droits, pour que des mesures soient prises, des lois votées par certains États dans les démocraties ${ }^{37}$. S'y sont pourtant maintenues sous des formes silencieuses, insidieuses, mais aussi ouvertes, connues de tous et jugées naturelles, des éléments insidieux ou tolérés d'une domination masculine à l'oeuvre dans le système patriarcal. (En Egypte par exemple,

36 Directrice de recherche au Centre National de la Recherche Scientifique (CNRS).

37 Ceux entre autres de S.de Beauvoir, Le deuxième sexe (1949). 
quel que soit le milieu, pauvre, modeste ou riche, un homme peut, en toute impunité, sans trop de risque, battre, brutaliser une femme dans l'espace domestique et, avec un parfait sentiment d'impunité, rendre un hommage appuyé à ses charmes, la toucher, se frotter contre elle dans l'espace public, - les transports en particulier -, sans que personne ne s'en alarme et $n$ 'intervienne, tant la situation est banale, quotidienne : les films « Les femmes du Caire " ou encore "Le bus 678 » le montrent parfaitement. Dans d'autres pays ce type de comportement sera moins toléré, voire dénoncé dans l'espace public, ce dont témoigne par exemple la création de parkings réservés exclusivement aux femmes en Allemagne, ou encore de brigades d'interventions dans les transports publics en France afin de repérer et sanctionner frotteurs, piqueurs, pinceurs. Tout ceci ne saurait empêcher, dans les pays où l'on dispose de chiffres, qu'une femme meure tous les trois jours sous les coups de son compagnon, qu'il s'agisse entre autres de l'Espagne ou de la France).

Combattus, mis en accusation, ces éléments de dépréciation, de dévalorisation et de violences infligées aux femmes semblent paradoxalement s'être intensifiés face aux menaces, aux attaques dont le système patriarcal fait à présent l'objet ${ }^{38}$. Une virilité virulente s'est en effet vue réaffirmée, en particulier avec l'arrivée au pouvoir de chefs d'état proclamant avec fierté un intérêt marqué et répété, portant exclusivement sur les attributs physiques des femmes, jeunes voire très jeunes. (Et ce de la part en particulier d'un Dutertre se désolant - à l'occasion de viols dont on l'informait - de ne pas avoir été le premier à violer; ou encore un Trump, affirmant savoir comment s'y prendre avec les femmes en les attrapant par leurs organes génitaux, Tout semble dit : mépris et réification d'un « sexe faible", usage et échange des femmes à titre d'objets sexuels par les hommes. Et pourtant, la nuit du 4 août 1789, nuit de l'abolition des privilèges en matière de naissance, de rang, avait voulu instaurer l'égalité pour tous les citoyens.

On s'inscrit ici dans une approche sociologique et historique voulant discerner la part de l'anthropologique et du politique dans l'inégalité entre

${ }^{38}$ HAROCHE, C. «La fabrique de la virilité dans la famille patriarcale ». Le journal des psychologues, n. 308, 2013. 
les hommes et les femmes. Certaines des observations visionnaires de Tocqueville portant sur les relations entre homme et femme, la comparaison qu'il en fait entre l'Amérique et la France, contribue à élucider en profondeur les relations entre hommes et femmes. On en vient ensuite à l'analyse de faits contemporains et on réexamine enfin en conclusion les causes lointaines et les formes - insidieuses en particulier - de cette dévalorisation persistante des femmes.

\section{Une abolition " partielle » des privilèges : la persistance du privilège masculin}

L'égalité des conditions suppose la considération, exige le droit au respect, à la dignité de tout individu quel qu'il soit, homme ou femme : le refus d'être méprisé, en raison entre autre de son identité sexuelle, le refus d'être disqualifié, enfermé dans une identité. Les revendications de liberté de choix en matière d'identité et d'orientation sexuelle, plus tard de genre, se sont faites jour dans les années 1990, des législations nouvelles allaient entre autre permettre des formes d'union entre personnes de même sexe : ces unions qui existaient depuis longtemps à l'abri des regards, font désormais - ou du moins tentent - de reconnaître le mariage pour tous, homosexuels aussi bien qu'hétérosexuels.

Posons nous à nouveau - à l'occasion de la reconnaissance de ces droits - la question de l'inégalité entre les hommes et des femmes, dans la sphère publique et sociale comme dans la sphère privée. $\mathrm{Y}$ a $\mathrm{t} \mathrm{il,}-\mathrm{y}$ aurait il - des privilèges insidieux, - insu-, qui tiendraient au sexe ? Et n'auraient pas été abolis tant ils sont tenus pour naturels ? Systémiques? Ces privilèges tiennent à la persistance du système patriarcal : et il s'agit là d'un impensé de la démocratie tant l'évidence d'un sexe fort dominant un sexe faible persiste dans l'histoire.

En quoi consiste cette inégalité ? Pour quelles raisons persiste-telle de façon continue quelles que soient les époques, les sociétés, les cultures, les traditions, les mœurs, les représentations mentales en matière de masculin et de féminin ? Les manières de se conduire, de concevoir le monde, les autres, soi-même que le système patriarcal impose ou induit tendraient à décliner dans un grand nombre de sociétés, en particulier dans les sociétés démocratiques contemporaines. S'y maintiennent toutefois, 
sous des formes implicites, insidieuses, mais souvent aussi explicites, des éléments de ce système.

Y perdure dans la longue durée l'idée même d'un « privilège masculin » tenant, comme le soulignaient Pierre Bourdieu (en 1998) et Françoise Héritier, à un système encourageant, imposant le « développement et (le) fonctionnement d'un moi masculin viril, fort ", relevant de l'amour de la mère vécue sur un mode fusionnel : l'amour sentimental exclusif pour la mère pourrait alors éclairer la mise à l'écart ultérieure de toute femme susceptible d'égaler cet amour initial.

Ce qui avait ainsi conduit Bourdieu à souligner que la féminité allait se réaliser essentiellement dans une fonction : la fonction maternelle, et au-delà, une fonction plus générale, " celle d'instituer des relations entre les hommes ". Les hommes y seraient acteurs de l'échange, pendant que les femmes seraient réduites à l'état d'objets et de signes d'échange s'inscrivant comme le dit également Bourdieu « dans un système d'alliance qui unit les hommes $»^{39}$. Françoise Héritier avait elle formulé l'existence d'un " modèle archaïque dominant » entendant par-là l'existence de stratégies de déconsidération, de dévalorisation des femmes considérées comme un objet ayant une valeur d'échange dans les relations entre hommes ${ }^{40}$.

La virilité, la force du masculin s'oppose à la faiblesse du féminin : le sexe dit faible aurait besoin de protection - qu'il s'agisse de la famille, du clan, de la tribu, en ce qui concerne le pouvoir, l'argent parfois, souvent en échange de sexe - face aux dangers et aux menaces extérieures. De ce point de vue le reportage montré à la télévision française sur les femmes voulant se retrouver dans un café proche de Paris, - dans le 93 pour être précis -, l'illustre parfaitement : seuls des hommes rébarbatifs, voire menaçants y sont installés, refusent de les servir, leur rappelant que leur place n'est pas au café : « ici c'est pas la France, c'est le bled ", lancentils. Tout est dit.

Par « privilège masculin » il faut entendre concrètement une liberté de se mouvoir à toute heure et en tout lieu, pendant que les femmes, elles,

39 BOURDIEU, P. La domination masculine. Paris : Éditions du Seuil, 1998.

40 HERITIER, F. (dir.). Hommes, Femmes. La construction de la différence. Paris : Le pommier/Cité des sciences et de l'industrie, 2005. 
se verraient souvent cantonnées à l'espace domestique, limitées dans cette liberté de mouvement par la crainte, la peur d'agressions pouvant aller du harcèlement psychique et physique au viol. Jugées dans leurs conduites, leurs façons de s'exprimer, de prendre la parole, dans leur mode de vie de manière générale, les femmes se voient au-delà et plus fondamentalement dévalorisées, dénigrées, disqualifiées, ce qui autorisent de la part de bien des hommes propos salaces, insultants, gestes déplacés. Fondamentalement la raison en serait que les hommes répondraient à la frustration ou au désir par la pulsion, pendant que les femmes savent se montrer dignes devant les pulsions de leurs compagnons, témoignent de retenue et de réserve, à moins de risquer d'être qualifiées d'hystériques.

Comment en est on toujours là, après la déclaration des Droits de I'homme (et pas comme on le souligne constamment de l'être humain, de l'individu, homme et femme) et du citoyen et surtout peut on en sortir? Et comment?

\section{"Exiger de la femme une abnégation d'elle-même »}

Revenons en premier lieu sur la persistance de l'inégalité entre homme et femme en dépit de l'avènement de la démocratie moderne : le statut de la femme est marqué par la dépendance dans les représentations, les conceptions qui la concernent. Cette dépendance répondrait à un besoin de protection. Or le besoin de protection ne saurait dans la démocratie relever d'un sexe et ne s'appliquer qu'à l'autre.

Tout aurait commencé en Amérique, soucieuse d'assurer l'égalité entre tous les citoyens, - en particulier entre hommes et femmes - dans une société, un pays qui tend à légiférer tous les rapports, des plus sociaux aux plus moraux et aux plus intimes. Ce qui on va le voir dans un instant concerne l'égalité des femmes aussi bien que des hommes.

Dès "l'Avertissement " de la Démocratie en Amérique Tocqueville confie ainsi en 1859 qu'il n'entend traiter que de l'égalité ${ }^{41}$ II se livre alors à une apologie des rapports qui tendent à reconnaître dans la démocratie

41 TOCQueVILLE, A. de. De la Démocratie en Amérique, t. 2. Paris : Garnier Flammarion, 1981. Voir aussi MILL, John Stuart. L'asservissement des femmes (1869). Paris : Petite Bibliothèque Payot, 2005. 
l'égalité entre "le fils et le père, le serviteur et le maître, et en général, l'inférieur et le supérieur ». Discernant un mouvement irrépressible vers l'égalisation des conditions Tocqueville souligne toutefois que les moeurs évoluent lentement, signalant qu'il faudrait «faire (de la femme) l'égale de l'homme ${ }^{42}$. Tocqueville semble en cela témoigner d'une pensée très moderne. Il se montre pourtant à cet instant " ......surpris et presque effrayé en voyant.... l'audace avec laquelle ces jeunes filles d'Amérique savaient conduire leurs pensées et leurs paroles", envisageant bientôt « les perils » que leur fait courir la Démocratie ${ }^{43}$. Il va alors concevoir « une espèce d'égalité démocratique » qu'il défınit comme une égalité dans des emplois différents, bien séparés. Et Tocqueville compare la situation des femmes en Amérique avec celle de la France où « il nous arrive souvent de donner aux femmes une éducation timide, retirée et presque claustrale, comme au temps de l'aristocratie ", pour les abandonner ensuite tout à coup, " sans guide et sans recours, au milieu des désordres inséparable d'une société démocratique ${ }^{44}$. Que peut-on en conclure ? Les femmes qui bénéficiaient de formes de protection - paternaliste certes - dans la famille patriarcale, se retrouveraient isolées et dépourvues de protections dans les institutions démocratiques.

Il observe à quoi l'éducation des filles les prépare en Amérique : après l'apprentissage de l'audace et de l'indépendance, vient celui de la dépendance silencieuse, l'acceptation du renoncement, l'enfermement dans un rôle, une fonction, l'apprentissage de l'indépendance de la jeune fille la prépare à être une bonne épouse et mère, mais pas à l'égalité, ajoutant "c'est d'elle-même et librement qu'elle se place sous le joug ", qu'elle se sacrifie, qu'elle renonce à ses ambitions professionnelles ${ }^{45}$.

Que faut il en conclure ? La société en Amérique gouverne les liens privés. Le juridique a pénétré jusqu'aux relations les plus intimes, tout ceci lui semble bien contraire à l'Europe où hommes comme femmes peuvent avoir des fonctions identiques, se soumettre aux mêmes devoirs,

42 TOCQUEVILLE. Ibidem, p. 263.

43 Ibidem, p. 248-249.

44 Ibidem, p. 248 et 263.

45 Ibidem, p. 252. 
et se voir reconnaître les « mêmes droits ". Ils se voient « mêlés en toutes choses, travaux, plaisirs, affaires ». Tocqueville conclut alors que loin de permettre de se réaliser selon leurs spécificités, l'absence de séparation, de division du travail des sexes conduisent à la situation en Europe où « II y a des gens ....qui, confondant les attributs divers des sexes, prétendent faire de l'homme et de la femme des êtres, non seulement égaux, mais semblables $»^{46}$.

\section{Persistance de privilèges, compensations financières, compensations psychologiques}

Pour ce qu'il en est des problèmes contemporains en matière de domination masculine insidieuse Tocqueville apparaît là encore comme prémonitoire : « aux yeux du législateur, la prostitution que la loi interdit clairement est bien moins à redouter que la galanterie ", que l'on ne saurait légiférer ${ }^{47}$.

Les américains ont voulu « donner à leurs différentes facultés un emploi divers; et ils ont jugé que le progrès ne consistait point à faire à peu près les mêmes choses à des êtres dissemblables, mais à obtenir que chacun d'eux s'acquittât le mieux possible de sa tâche ". II s'agit là clairement d'une division du travail entre sexes. Et c'est la raison pour laquelle ils ont tout naturellement pensé au nom des affaires, du commerce, de son organisation « que toute association, pour être efficace, devrait avoir un chef, et que le chef naturel de l'association conjugale était I'homme " Ils lui reconnaissaient « le droit de diriger sa compagne; et ils croient que, dans la petite société du mari et de la femme, ainsi que dans la grande société politique, l'objet de la démocratie est de régler et de légitimer les pouvoirs nécessaires, et non de détruire tout pouvoir ${ }^{48}$. Le système patriarcal vient ainsi appuyer le fonctionnement du couple que Tocqueville considère comme une association à des fins d'organisation sociale et économique.

\footnotetext{
46 Ibidem, p. 263.

47 Ibidem, p. 259.

48 Ibidem, p. 264.
} 
Tocqueville souligne enfin «Aux État-Unis, on ne loue (flatte) guère les femmes; mais on montre chaque jour qu'on les estime ". Mais les choses sont pourtant loin d'être aussi claires : "ils montrent une même estime pour le rôle de chacun d'eux, et les considèrent comme des êtres dont la valeur est égale». Mais guère leur place, leur fonction dans la hiérarchie de la société. De même qu'en Europe et davantage encore « on a remarqué souvent.....(qu') un certain mépris se découvre au milieu même des flatteries que les hommes prodiguent aux femmes : bien que l'Européen se fasse souvent l'esclave de la femme, on voit qu'il ne la croit jamais sincèrement son égale " alors "on les considère comme des êtres séduisants et incomplets » : des êtres infantiles, plutôt qu'infantilisés. Tocqueville va alors tout aussitôt remarquer que « les femmes elles mêmes..... ne sont pas éloignées de considérer comme un privilège la faculté qu'on leur laisse de se montrer utiles, faibles et craintives $"{ }^{49}$. Sous estimées mais protégées par un homme, c'est en cela peut être que certains pourraient aller jusqu'à penser qu'il s'agit là d'un privilège féminin ".

Les américains manifestent donc du respect mais se garderaient de tout comportement de séduction empressée : ils ne sauront pourtant éviter les plaintes pour harcèlement et viol. Le modèle féminin de la retenue et de la réserve garantit à la femme la dignité, - qu'on requiert et qu'on approuve : c'est à dire la conscience de leur place, l'acceptation de leur sujétion, leur soumission à l'homme, leur silence. Les femmes témoignent d'une absence de pulsions : elles se définissent par la retenue, le refoulement. La séduction et le harcèlement sont réservés aux hommes.

Tocqueville en vient enfin aux législateurs et compare "les législateurs des États-Unis, qui ont adouci presque toutes les dispositions du Code Pénal, à l'exception du viol qu'ils punissent de mort; et il n'est point de crimes que l'opinion publique poursuive avec une ardeur plus inexorable [...] En France, où le même crime est frappé de peines beaucoup plus douces, il est souvent difficile de trouver un jury qui condamne $»^{50}$.

Libéral, conservateur, Tocqueville, on le voit, était un visionnaire : certains éléments de ses analyses ne sauraient ainsi être négligés par

49 Ibidem, p. 265-266.

50 Ibidem, p. 266. 
les analyses contemporaines pour saisir leurs causes profondes relevant d'éléments d'un système patriarcal, d'une conception de la famille la tenant pour protectrice profondément ancré dans les traditions : ces éléments pourraient expliquer l'enfermement dans un rôle de dépendance et de sujétion invitant à faire l'hypothèse d'un système de compensations financières, respectant le puritanisme et assurant le commerce comme valeur suprême.

\section{Les hommes, des sujets sexuels. Les femmes, des objets sexuels}

Qu'est ce qui aurait changé depuis les observations et les analyses de Tocqueville ? Les modes de diffusion de l'information, la circulation des images, les modes de relations que les individus peuvent instaurer entre eux. À distance, dans le travail, par les réseaux sociaux. Une information désormais globale et immédiate nourrie (parfois) d'expériences vécues et désormais partagées dans un grand nombre de mouvements. Ce qui est inédit c'est la participation - à un mouvement, un engagement - , au titre d'un vécu individuel dans des sociétés individualistes par le biais des réseaux sociaux. Avoir ainsi éprouvé une dévalorisation sous une forme ou une autre due entre autre au fait que l'on est une femme et en partager l'expérience - dans le cas du mouvement « \# me too » : un tel mouvement extraordinaire, moteur d'engagement, pourra effrayer un gouvernement, autoritaire en particulier, voire tout gouvernement.

Le terme communauté a un sens plus incertain et plus imprécis que du temps de Tocqueville alors largement déterminé par l'espace. On se trouve face à des communautés d'un nouveau genre : des modes de résistance à un état donné de société que des individus, des groupes, des populations souhaitent faire progresser en obtenant de nouveaux droits, ou en voulant le rétablissement de droits antérieurs, de libertés publiques dans un régime totalitaire. Il peut s'agir là également de communautés pouvant être menaçantes dans la mesure où elles sont susceptibles d'être porteuses d'incitations à une grande violence, à la destructivité la plus extrême : ces communautés constituent en effet souvent des regroupements d'individus (voulant rester) anonymes, ou d'associations partageant des valeurs identiques. 
Auraient ainsi perduré des éléments implicites d'un système patriarcal. Les femmes se plaignent de l'inégalité de traitement dans tous les sens du terme - économique, sociale, psychologique et psychique entre hommes et femme, d'une domination masculine continue, insidieuse. Ayant leur place, leur fonction dans l'espace public, social, les hommes sont - ou du moins savent se montrer - à l'aise en société, sérieux ou drôles. Les femmes, à l'inverse, ont tout à prouver, leur intelligence d'abord, leur compétence, elles sont vite considérées comme des bas bleus ennuyeuses et frustrées. On leur reprochera certaines de leurs attitudes en déduisant qu'elles sont lourdes, paranoïaques, qu'elles sont mal à l'aise, intimidées, embarrassées, qu'elles ne comprennent pas la plaisanterie ${ }^{51}$.

Bourdieu avait pris des exemples dans la sphère du travail, dans l'entreprise en particulier en France. Organisations où un patron "presque toujours un homme, exerce une autorité paternaliste, fondée sur l'enveloppement affectif ou la séduction » ajoutant encore " surcharge de travail et prenant en charge tout ce qui se passe dans l'institution, (il) offre une protection généralisée à un personnel subalterne principalement féminin $»^{52}$. Autre scène professionnelle, dans un atelier de sous-traitance automobile: Sophie fait le récit suivant à propos du responsable : «les garçons, il les forme sans engueulade. Il y a avec eux comme le cérémonial du compagnon avec son élève ». Le patron convoque un beau jour le responsable en question. On m'a rapporté que « vous traitez très souvent Sophie de blonde. Pourquoi ? " Réponse : par " gentillesse ", pour éviter la brutalité, ne pas la traiter de crétine « à longueur de journée ». Donc, reprend le patron, c'est bien une sorte d'insulte que vous proférez à son égard ». En aucun cas : s'il la traite de "blonde ", " c'est qu'elle a un coté juvénile, elle paraît délicate, si fragile ». "Elle n'est pas fragile », réplique le patron. "C'est vous qui la fragilisez avec cette dévalorisation permanente ». Elle est parfaitement qualifiée, compétente, il convient juste de lui apprendre " comment faire sans l'engueuler ». Autre scène professionnelle, lors

\footnotetext{
51 Il faut ici noter - et c'est très révélateur - qu'il y a aujourd'hui beaucoup de femmes excellentes humoristes en France.

52 Bourdieu apud C. Haroche, Histoire de la virilité. Paris : Seuil, 2011.
} 
d'un repas. Un homme jovial, convivial, sauf avec une femme ayant un poste important. « Jamais, pourtant elle ne parvient à capter son regard. La moindre remarque, le moindre rire " sont adressées à "son adjoint " jamais à elle ${ }^{53}$. Déqualification subreptice. Elle n'existe pas.

D'autres formes de disqualification insidieuse sont à l'oeuvre dans la duplicité : fausse courtoisie, cordialité appuyée, excès de politesse. Ainsi d'un « Madame ", prononcé avec insistance : on s'adresse à la femme en l'excluant subtilement, ou du moins en ignorant sa qualité professionnelle, on lui rappellent son statut de femme, et on l'y réduit. Ainsi encore de l'usage du vouvoiement envers les femmes dans les cercles professionnels ou sociaux où l'on se tutoie par esprit de corps. Autre lieu, mêmes moeurs. Une réunion au ministère des Finances, à propos d'une entreprise en difficulté : parmi les participants une seule femme. Elle observe, elle aussi, que « depuis son arrivée, il y a trois mois [...] on la regarde sans la voir. C'est comme si elle était transparente ". Elle remarque encore que l'un des responsables « est toujours très correct avec elle [...] un air, un brin cérémonieux ". L'objet de cette réunion : la lutte contre la concurrence asiatique, « la réduction de la marge sur les machines à laver». C'est le moment qu'il choisit pour se touner vers elle pour la première fois, et la prendre à témoin : "Car les ménagères ne s'en laissent plus conter. Vous savez bien sûr de quoi je parle Madame ${ }^{54}$.

Dans un essai récent, Laure Murat s'interroge sur le fait de savoir si « le mouvement '\# me too' déclenché par l'affaire Weinstein va provoquer un changement durable et en profondeur de la société ${ }^{55}$ ? Elle rappelle après Bourdieu et Héritier que « la pratique est millénaire........les femmes savent, dès l'enfance le plus souvent, qu'elles sont assimilées à des objets sexuels. Et ce savoir là est universel ». Elle en conclut « où qu'elles soient « les femmes (sont) en danger »: dans la famille, l'entourage, avec l'inceste et peu protégées isolées dans la démocratie. Murat remarque que

\footnotetext{
53 Ibidem.

54 Ibidem.

55 Ibidem.
} 
ce n'est que « très tardivement (que le harcèlement a été) isolé et identifié juridiquement $»^{56}$. Alors que le problème est très ancien.

Les frontières entre séduction et harcèlement, outre le fait qu'elles ne sont pas toujours claires, ne sont pas perçues, tolérées et sanctionnées de la même façon en Amérique et en France. En ce qui concerne la France Tocqueville parlait de galanterie et de séduction, de ce qui s'abrite sous le terme séduction, et ce que permet celui de galanterie, rabaisser en flattant outrageusement. Et c'est précisément sur ce dernier point que nous voulons nous centrer.

Les luttes féministes se sont dressées contre la toute puissance du système patriarcal. Quelle réponse donner au harcèlement insidieux ? Quelle réponse donne-t-on à la domination masculine, comment non pas la supprimer, mais la diminuer considérablement selon qu'on est en Amérique ou en France?

Laure Murat va opposer à l'instar de Tocqueville un siècle et demi auparavant la galanterie française au puritanisme américain soulignant que « politiquement, juridiquement la femme a été mineure et est restée dévalorisée ${ }^{57}$. Elle est exclue des décisions sérieuses. Sa force elle l'exercera sur le plan privé dans la sphère privée.

\section{Argent ou Galanterie?}

La galanterie s'inscrivant dans la « tradition » repose sur une certaine conception des rapports homme-femme ce que la femme peut donner dans le registre domestique du ménage et des faveurs, (une femme galante étant une prostituée). La galanterie dans la mesure où elle est uniquement réservée aux femmes apparaît comme un système de compensations durable correspondant à un enfermement dans le féminin et la faiblesse et en aucun cas de protection. "Pourquoi la haine, le mépris, l'avilissement des femmes sont ils des thèmes aussi populaires ${ }^{58}$ ? Ne faudrait il pas

56 MURAT, L. Une révolution sexuelle? Paris: Stock, 2018.

57 Ibidem.

58 Ibidem. 
voir une première raison à ce mépris continu dans la peur de la faiblesse, de l'impuissance?

Pourquoi le sexisme et le machisme? Ce mode de comportement instaure incontestablement des liens entre les hommes, permettant de développer une complicité entre hommes cimentée contre les femmes.

Tocqueville trouve également à s'éclairer grâce aux écrits de Freud sur l'agressivité et aux travaux récents que Laurence Kahn leur a consacrés instaurant un dialogue par moments lumineux avec les écrits Freudiens ${ }^{59}$. S'interrogeant sur les causes du Malaise dans la civilisation, Freud a souligné « la déficience des dispositifs qui règlent les relations des hommes entre eux dans la famille, l'Etat et la société » II faut en conclure que «l'homme ne supporte pas les limites, la culture au sens large incluant le droit. " Et c'est précisément la difficulté à laquelle se heurte de façon continue le mouvement "\# me too » : les droits ont toujours à lutter contre les traditions et les coutumes qui les bafouent dans nombre de cultures. Ils ne sont jamais définitivement acquis : constamment menacés ils n'ont jamais pu empêcher les régressions. Le besoin de protection et de réconfort dont tout individu a besoin est assuré par le mot «culture » qui suppose des limites et désigne « la somme totale des dispositifs..... qui servent à deux fins : la protection de l'homme contre la nature et la réglementation des relations des hommes entre eux". Limiter la force physique individuelle par des règles, des droits qui relèvent des institutions juridiques, l'individu homme ou femme a d'abord été " plein de faiblesse ", « un nourrisson en désaide » qui se verra conférer de la puissance par la protection de la famille et des institutions.

Il s'agit d'une nécessité absolue : « si une telle tentative n'avait pas lieu, ces relations seraient soumises à l'arbitraire de l'individu, c'est à dire que le plus fort physiquement en déciderait dans le sens de ses intérêts et motions pulsionnelles ». Existe une compétition physique mais plus encore psychique entre hommes. "Selon nous aucun autre trait ne caractérise mieux la culture que l'estime et les soins accordés aux activités psychiques supérieures, aux performances intellectuelles, scientifiques et artistiques,

59 KAHN, Laurence. Fictions et vérité freudienne : Entretiens avec M. Einaudeau. Paris : Les Belles Lettres, 2018. 
au rôle directeur concédé aux idées dans la vie des hommes " et ce serait très précisément ce dont les femmes sont dépourvues et c'est tout cela qui n'est pas reconnu aux femmes, qui leur est dénié. C'est cela qui a changé depuis Tocqueville et Freud : la prise de conscience, - l'explicitation de la vulnérabilité de chacun, la nécessité des protections.

\section{L'impossibilité de légiférer l'agressivité insidieuse}

Freud avait posé que « la vie en commun des hommes n'est rendue possible qui si se trouve réunie une majorité qui est plus forte que chaque individu » : il était convaincu que "la puissance de cette communauté (s'opposait) maintenant en tant que 'droit' à la puissance de l'individu qui est condamnée en tant que "violence brute ». Il avait ainsi vu dans "ce remplacement de la puissance de l'individu par celle de la communauté » la certitude du « pas culturel décisif ». Son raisonnement tenait à ce que "les membres de la communauté se (limitaient) dans leurs possibilités de satisfaction, alors que l'individu isolé ne connaissait pas de limite de ce genre ". Si Freud semblait confiant dans l'étape suivante "celle de la justice, c'est à dire l'assurance que l'ordre de droit, une fois donné, ne (serait) pas de nouveau battu en brèche en faveur d'un individu » il avait pourtant décelé dans le malaise dans la culture, un malaise renforcé dans le droit qui fait partie de la culture car « rien (n'était) décidé sur la valeur d'un tel droit ". On observe, on pressent, on décèle les tendances profondes à l'oeuvre dans la société mais que l'on ne peut prévoir ni maitriser précisément, les irruptions de l'agressivité insidieuse et l'impossibilité de la légiférer, ce que reconnaissait Freud.

Laurence Kahn revient, se référant aux travaux de Vernant, aux fondements mêmes de la démocratie dont la constitution va de pair avec « le premier cercle d'égalité de la parole ": c'est là qu'elle décèle le caractère tragique de la démocratie « qui exige en effet une « formidable limitation pulsionnelle ${ }^{60}$. Laurence Kahn contribue à la compréhension en profondeur entre autre du mouvement « \# me too " en se penchant sur la tendance à l'agression qui apparaît comme une exigence de limitation des pulsions. Freud, remarque-t-elle, a réfléchi très longuement sur «les

60 KAHN, Laurence. Fictions et vérité Freudienne, op.cit. p. 165. 
systèmes de limitation pulsionnelle " y voyant " la base même de la culture $»^{61}$.

Kahn rappelle la destructivité à l'oeuvre en tout individu, en tout groupe et entre groupes, la peur d'être dépourvu de protection et en conséquence la peur de ne pas ou plus s'appartenir. Or la culture suppose le refoulement pulsionnel, en conséquence l'acceptation de la limitation qui prévient la violence, mais qui n'assure pas nécessairement la protection de la faiblesse, de la vulnérabilité, de l'impuissance.

Laurence Kahn s'efforce de comprendre " ce qui, au dedans de chaque individu, quel que soit ce qu'on appelle le progrès de la civilisation, impulse très régulièrement à nouveau la haine absolue de l'autre, comme ennemi, comme différent, comme étranger à soi " ${ }^{62}$. Et cet autre c'est en particulier la femme, les femmes de manière générale excepté la mère dans le patriarcat. Kahn rappelle que «l'agression est constamment au centre de la pensée de Freud..... sous la forme de l'irruption de la pulsion sexuelle dans la vie psychique, et sous la forme de la violence du désir et de ses conséquences " ce qui expliquerait cette violence continue à l'endroit des femmes ${ }^{63}$.

En reprenant et commentant Freud, Laurence Kahn permet peut être d'aller au delà : elle fait en effet très justement remarquer que «Freud ne cherche pas l'origine de la barbarie....dans les transformations historiques des sociétés ${ }^{64}$. Freud cherche l'origine de la barbarie " dans la configuration intérieure de tout individu » dans une perspective anthropologique et clinique trouvant dans la peur de la séparation originelle, dans celle de l'abandon, une explication à l'angoisse existentielle ${ }^{65}$.

L'enseignement de Freud incite à formuler, « à créer des compromis » : ceux ci devraient "permettre, j'ai presqu'envie de dire " confie ainsi Laurence Kahn, "de vivre avec cette barbarie ". "Toutes les sociétés procèdent d'un système, d'un dispositif qui se situe à la charnière, au

\footnotetext{
61 Ibidem, p. 163

62 Ibidem, p. 181

63 Ibidem, p. 179

64 Ibidem, p. 181

65 Ibidem.
} 
Carrefour entre l'horreur et le renoncement ${ }^{66}$ Peut-on dire que l'horreur, la barbarie pourrait aujourd'hui tenir aux effets - renforcés - d'une illimitation générale des pulsions induisant le refoulement et au delà le renoncement?

La mise en garde de Freud est très claire «si on refoule (cette agressivité) purement et simplement les retours ne seront que plus dévastateurs ${ }^{67}$.

\section{En conclusion}

Dans une synthèse d'une grande élégance, Laurence Kahn souligne la nécessité de trois systèmes, « premièrement de protection, deuxièmement de limitation, troisièmement de dérivation ${ }^{68}$.

Un système de protection et de limitation se met en place progressivement et ne se défait que très progressivement, partiellement et pas complètement. Mais un tel système peut toujours se durcir, les droits ne sont jamais défınitivement acquis.

Le besoin de protection - qu'il s'agisse des hommes comme des femmes - fait partie des besoins humains, des besoins psychiques : il fait partie des droits et pourtant il semble ne se poser essentiellement que pour les femmes.N'y aurait il pas un lien obscur entre protection masculine et ce que certains dans la tradition - libérale patriarcale -pourraient considérer comme « privilège féminin » ? "L'idée de Freud est toujours que la culture permet de créer de plus grandes unités humaines, de rassembler..... ": permettrait elle de dépasser cette " hostilité primaire qui ne se dément jamais dans le devenir de l'humanité ${ }^{69}$ ? Est-on à présent confronté au dépassement de la tribu et de la communauté vers la société, vers l'état démocratique comme le pensait Freud ? Ou à une régression tribale, à un tribalisme généralisé dans lequel on affaiblit les femmes en les ramenant

\footnotetext{
Ibidem, p. 182, p. 184

Ibidem, p. 182

Ibidem, p. 182.

69 Ibidem, p. 185 et 199.
} 
à la vulnérabilité de leur corps et de leur psyché ? On les protège alors en les étouffant, en les enfermant.

C'est précisément ce que fait remarquer récemment Françoise Héritier «il ressort des expériences historiques et ethnologiques que la force seule n'a jamais suffi à maintenir des dominés en situation de dépendance - sauf à recourir à l'extermination - si elle ne s'accompagne d'autres rouages de l'oppression qui sont de nature idéologique ${ }^{70}$.

Dans toute domination existe une part - difficile à mesurer - de servitude, parfois volontaire, souvent inconsciente de soumission, d'asservissement, et surtout de peur : s'y soustraire prend du temps, nécessite une lente maturation, elle n'est pas impossible....

\section{Bibliographie}

BOURDIEU, P. La domination masculine. Paris : Éditions du Seuil, 1998.

HAROCHE, C. «La fabrique de la virilité dans la famille patriarcale ». Le journal des psychologues, n. 308, 2013.

HÉRITIER, F. (dir.). Hommes, femme : La construction de la différence. Paris : Le Pommier/Cité des sciences et de l'industrie, 2005.

KAHN, L. Fictions et vérité freudienne : Entretiens avec M. Einaudeau. Paris : Les Belles Lettres, 2018.

MILL, J. S. L'asservissement des femmes (1869). Paris : Petite Bibliothèque Payot, 2005.

MURAT, L. Une révolution sexuelle? Paris : Stock, 2018.

TOCQUEVILLE, A. De la democratie en Amérique. t. 2. Paris: GarnierFlammarion, 1981.

70 HÉRITIER, F. (dir.). Hommes, femme : La construction de la différence. Paris : Le Pommier/Cité des sciences et de l'industrie, 2005. 